\title{
Formulation and characterization of nanofibers and films with carvedilol prepared by electrospinning and solution casting method
}

\author{
Marko Krstić ${ }^{\mathrm{a}, *}$, Marija Radojević ${ }^{\mathrm{a}}$, Dušica Stojanović ${ }^{\mathrm{b}}$, Vesna Radojević ${ }^{\mathrm{b}}$, Petar Uskoković ${ }^{\mathrm{b}}$, Svetlana Ibrić $^{\mathrm{a}}$ \\ a University of Belgrade - Faculty of Pharmacy, Department of Pharmaceutical Technology and Cosmetology, Vojvode Stepe 450, 11221 Belgrade, Serbia \\ b University of Belgrade - Faculty of Technology and Metallurgy, Department of Materials Science and Engineering, Karnegijeva 4, 11120 Belgrade, Serbia
}

\section{A R T I C L E I N F O}

\section{Article history:}

Received 25 July 2016

Received in revised form 11 January 2017

Accepted 5 February 2017

Available online 6 February 2017

\section{Keywords:}

Nanofibers

Oral films

Dissolution rate

Process parameters

Formulation parameters

Poly (ethylene oxide)

\begin{abstract}
A B S T R A C T
The preparation and characterization of films and nanofibers with carvedilol as a poorly water-soluble drug in poly (ethylene oxide) (PEO) polymer were investigated. Films are prepared by solution casting method, and nanofibers by electrospinning from a polymer solution. Water and mixture of ethanol and water were used as solvents. FT-IR analysis of the samples showed that there was no interaction between the polymer and the drug substance. DSC analysis revealed that carvedilol was dissolved in the polymer and influenced the degree of crystallinity of PEO. Carvedilol release rate for all of the formulations was increased in comparison with pure carvedilol. Significant differences in the rate of release of carvedilol from the films and nanofibers were observed. Field Emission Scanning Electron Microscope (FESEM) images of the obtained fiber was revealed the dependence of the fiber diameter of formulation and electrospinning process parameters, and consequently influence the amount and distribution of carvedilol in the encapsulated fibers.
\end{abstract}

@ 2017 Elsevier B.V. All rights reserved.

\section{Introduction}

Special attention in recent years is given to the formulation development of poorly soluble drugs, since dissolution of poorly soluble drugs is the rate limiting step for drug absorption and bioavailability (Löbenberg and Amidon, 2000). A number of alternative technologies have been developed to overcome the drawbacks associated with poor aqueous solubility; electrospun nanofibers and oral films are promising ones (Bertoncelj et al., 2014; Siemann, 2005).

Electrospinning is a versatile technique through which a variety of constructs can be obtained with application in biomedicine (medical prosthesis, tissue scaffolds, wound dressings, drug delivery, cosmetics), textiles, electricity and optics, sensors, filtration, catalysis, unconventional energy sources and storage cells (Hunley and Long, 2008; Huang et al., 2003). In the field of drug delivery and tissue engineering, electrospun polymer nanofibers have gained increasing importance because they present several advantages: relatively easy drug entrapment during the electrospinning process, obtaining of high loadings if so desired, burst control, stability and preservation of drug/growth factor activity, high surface area to volume ratio (which enhances drug release) and specific morphology which can be easily controlled during the electrospinning process (Agarwal et al., 2008). The polymer fibers are formed from a solution between two electrodes bearing electrical charges of opposite polarity. One of the electrodes is placed into the

\footnotetext{
* Corresponding author.

E-mail address: mkrstic109@gmail.com (M. Krstić).
}

solution and the other onto a collector. Once ejected out of a metal needle, the charged solution of the jets evaporates to become fibers, which are collected on the collector. The structure and the morphology of electrospun polymer materials, be it fibers or particles, are determined by the synergistic effects of solution parameters (polymer concentration, solution viscosity and flow rate) and electrostatic forces (applied voltage and distance between the needle and collector) (Dukali et al., 2014). Immediate or modified drug release can be achieved by the selection of a polymer for nanofiber production and the manner of the drug loading. The drug can be either incorporated into the polymer matrix of the nanofibers or bound to their surfaces (Huang et al., 2003; Meinel et al., 2012; Rošic et al., 2012). Factors that can affect the drug release from electrospun fibers are: fiber construct geometry and thickness (Okuda et al., 2009), fiber diameter and porosity (Cui et al., 2006; Bertoncelj et al., 2014; Sill and von Recum, 2008), fiber composition (Buschle-Diller et al., 2007), fiber crystallinity (Kenawy et al., 2002), fiber swelling (Xie and Buschle-Diller, 2010), drug loading (Cui et al., 2006; Xie and Buschle-Diller, 2010), drug state (Zamani et al., 2010; Xie and Wang, 2006), drug molecular weight (Buschle-Diller et al., 2007; Taepaiboon et al., 2006), drug solubility in the release medium (Xie and Buschle-Diller, 2010), drug-polymer-electrospinning solvent interactions (Chew et al., 2005; Zeng et al., 2005).

The solution casting is one of the oldest methods of film production. This method has been later replaced with extrusion, pressing and polymer blowing from the melt. However, it is still used for preparation of high quality thin films, especially if combined with the spin coating method for application to the substrates. The following conditions 
Table 1

Composition of films (F1-F3) and nanofibers (F4-F10), preparing method, collector surface areas and flow rate (PEO - poly (ethylene oxide); SC - solution casting; ES electrospinning).

\begin{tabular}{|c|c|c|c|c|c|c|c|c|c|c|}
\hline Formulation & $\begin{array}{l}\text { F1 } \\
\text { Filn }\end{array}$ & & F3 & $\begin{array}{l}\mathrm{F} 4 \\
\text { Nano }\end{array}$ & $\begin{array}{l}\text { F5 } \\
\text { ofibers }\end{array}$ & F6 & F7 & F8 & F9 & F10 \\
\hline $\begin{array}{l}\text { Carvedilol, with respect } \\
\text { to PEO, \% }\end{array}$ & 5 & 10 & 10 & 5 & 10 & 10 & 10 & 10 & 10 & 10 \\
\hline Solvent I: Water & + & + & & + & + & & & + & + & + \\
\hline $\begin{array}{l}\text { Solvent II: } \\
\text { Ethanol/Water } \\
(40 / 60, \mathrm{w} / \mathrm{w})\end{array}$ & & & + & & & + & + & & & \\
\hline Preparing method & SC & SC & SC & ES & ES & ES & ES & ES & ES & ES \\
\hline $\begin{array}{l}\text { Collector surface area } \\
\left(\mathrm{cm}^{2}\right)\end{array}$ & & & & 121 & 121 & 121 & 121 & 1156 & 1156 & 1156 \\
\hline Flow rate, $\mathrm{ml} / \mathrm{h}$ & & & & 1 & 1 & 0.2 & 0.5 & 0.2 & 0.5 & 1 \\
\hline
\end{tabular}

must be met for this method: the polymer must be soluble in a liquid solvent or in water; a solution with the appropriate viscosity should be formed; a homogenous film, easily extractable from the mould should be formed (Siemann, 2005).

Carvedilol, nonselective beta blocker, was used as a model drug. It is poorly soluble in water $\left(0.583 \mathrm{mg} / \mathrm{l}\right.$ at $\left.25^{\circ} \mathrm{C}\right)$ and highly permeable, belonging to the BSC class II (Kalimuthu and Yadav, 2009). So far, carvedilol nanofibers, successfully developed by different polymers, have been described in literature. Nanofibers obtained by electrospinning from carvedilol solutions were obtained using following polymers: poly(butyl methacrylate-co-(2-demethylaminoeethyl) methacrylateco-methyl methacrylate) - Eudragit ${ }^{\circledR} \mathrm{E}$, poly(methacylic acid-co-methyl methacrylate) - Eudragit ${ }^{\circledR}$ L 100-55, polyvinylpyrrolidone K90 (Balogh et al., 2015a, 2015b), polycaprolactone (Potrč et al., 2015), vinylpyrrolidone-vinyl acetate copolymer - Kollidon ${ }^{\circledR}$ VA 64 (Balogh et al., 2015a, 2015b). Carvedilol - Eudragit ${ }^{\circledR}$ EPO (poly(butyl methacrylate-co-(2-dimethylaminoethyl) methacrylate-co-methyl methacrylate) $1: 2: 1$ ) nanofibers, prepared by melt electrospinning were described in literature by (Balogh et al., 2014; Krist of ' Nagy et al., 2013). The solid dispersion methods for BCS class II drugs were developed as pharmaceutical forms in which a drug is dispersed in an inert matrix. These hydrophilic matrices, also called "carriers" form the backbone of solid dispersion formulations increase the solubility and dissolution profile of hydrophobic drugs by several mechanisms, such as increased porosity, reduction in agglomeration and particle size, improved wettability and solvent access due to increased surface area. Good results were obtained with Polyvinylpyrrolidone K30 and Eudragit ${ }^{\circledR}$ L100 (Yuvaraja and Khanam, 2014 and Lee et al., 2013.). In this study the presence of poly (ethylene oxide) (PEO) and Polysorbat 80 increased solvatation (dissolution) of carvedilol by surrounding of drug molecules with hydrophilic molecules of PEO and enhancing wettability of drug molecule (Dewan et al., 2012; Hammouda, 2006 and Balogh et al., 2014). In the other manner the hydrophobic parts of PEO also tend to attract the similar part from carvedilol molecules. A drug is thoroughly dispersed in a water-soluble carrier.

The aim of this work was formulation, development and evaluation of nanofibers and oral films, as potential carriers for carvedilol, produced from PEO polymer solutions. Carvedol/PEO solutions, prepared in water or ethanol/water mixture was casted to produce films, or electrospun to produce nanofibers. In electrospinning process, flow rate and collector surface area were varied. Produced films and nanofibers were evaluated with respect to drug content, degree of crystallinity and drug release. Morphology of nanofibers was also observed. Finally, formulation with higher drug content (compared to declared) and the fastest drug release will be chosen.

\section{Materials and methods}

\subsection{Materials}

Carvedilol, which was used as a model substance, was Ph. Eur. 8 grade. Polysorbate 80 (Sigma-Aldrich Chemie GmbH, Germany) was used as a surfactant. Poly (ethylene oxide) (PEO) with a molecular weight of $600,000 \mathrm{~g} / \mathrm{mol}$ (Acros Organics, Belgium) was used as a polymer. Ethanol ( $99.5 \mathrm{v} / \mathrm{v})$, which was Ph. Eur. 8 grade, was used as solvent and deionized water (DI) (resistance of $18 \mathrm{M} \Omega \mathrm{cm}$ ) was used for the preparation of solutions.

\subsection{Methods}

\subsubsection{Preparation of nanofibers and films}

The two series of the PEO solutions for electrospinning and solution casting; were prepared: one was PEO in water; second PEO in ethanol/ water mixture $(40 / 60, w / w)$. Firstly, the amount of $1 \mathrm{~g}$ of PEO was added in $19 \mathrm{ml}$ of deionized water and stirred $12 \mathrm{~h}$. In this solution $0.1 \mathrm{~g}$ $(0.05 \mathrm{~g})$ carvedilol and $0.02 \mathrm{ml}$ of Polysorbat 80 were added and mixed continuously for $24 \mathrm{~h}$. The same procedure was used for solution prepared with solvent consisted of ethanol/water mixture (40/60, w/ w). The complete solubility of carvedilol was achieved as a consequences of the presence of PEO and Polysorbat 80 in the water (Dewan et al., 2012; Hammouda, 2006; Balogh et al., 2015a, 2015b; Yang et al., 2015).

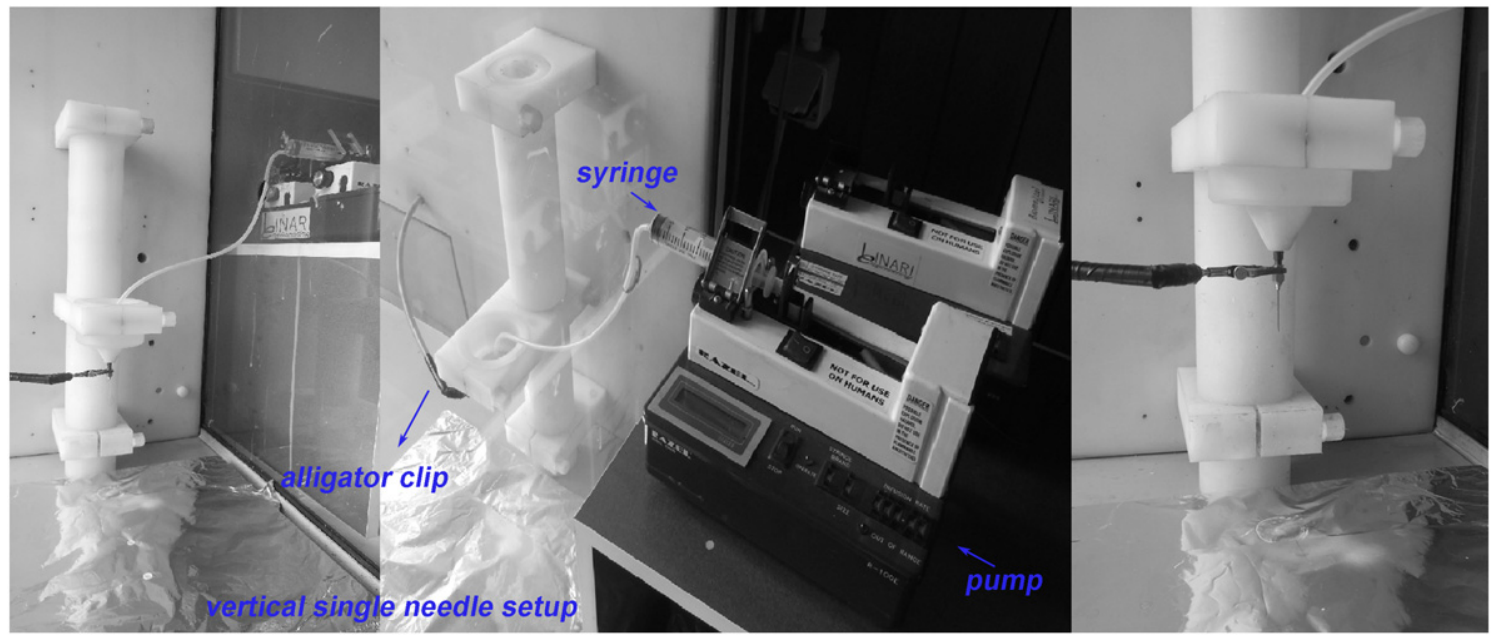

Fig. 1. Photographs of electrospinning setup. 
Table 2

Results of carvedilol assay in formulations F1-F10 presented as percent of declared content.

\begin{tabular}{|c|c|c|c|c|c|c|c|c|c|c|}
\hline Formulation & $\mathrm{F} 1$ & F2 & F3 & $\mathrm{F} 4$ & F5 & F6 & F7 & F8 & F9 & F10 \\
\hline Content (\%) & 92.80 & 96.02 & 95.30 & 98.40 & 92.40 & 45.70 & 47.00 & 90.24 & 89.21 & 86.32 \\
\hline
\end{tabular}

Prepared solutions was used both for electrospinning (nanofibers) and for solution casting (film).

Electrospinning process (Electrospinning setup $\mathrm{CH}-01$, Linari Engineering, Italy) was setup as follows: a $20 \mathrm{ml}$ plastic syringe with a metallic needle of $1 \mathrm{~mm}$ inner diameter was set vertically with the syringe pump (R-100E, RAZEL Scientific Instruments); the high-voltage power supply (Spellman High Voltage Electronics Corporation, Model: PCM50P120) was set to the voltage of $28 \mathrm{kV}$. Distance from the needle tip and collector was $15 \mathrm{~cm}$. The grounded copper collector, rectangular shape, overlaid with aluminum foil, was used for collecting nanofibers. Based on the screening studies, two process parameters, the surface area of collector and flow rate, were chosen and varied. Two collectors of different surface area were used: $121 \mathrm{~cm}^{2}$ and $1156 \mathrm{~cm}^{2}$ (Table 1). The flow rate of the solution was adjusted according to the formulation (Table 1). The electrospinning solution was spun in the single needle setup. All equipment was setup in closed chemical laboratory draft chamber system ventilated cabinet (Fig. 1).

For the solution casting method, prepared solutions were poured into flat-bottomed Petri dishes and dried $48 \mathrm{~h}$ at room temperature.

\subsubsection{Carvedilol assay in nanofibers and films}

Carvedilol assay in formulations was done in order to assess amount of carvedilol retained in nanofibers and films. The exact amount of nanofibers/films was dissolved in specified amount of $0.1 \mathrm{M} \mathrm{HCl}$. Carvedilol content was evaluated with UV spectroscopy, on $241 \mathrm{~nm}$, using UV/VIS spectrophotometer Evolution 300 (Thermo Fisher Scientific, Cambridge, UK). Carvedilol content was expressed in percent, as the ratio between carvedilol content determined in formulation and declared content of carvedilol. The results were shown as medium value of three repeated measurements for each sample.

\subsubsection{Field Emission Scanning Electron Microscope (FESEM)}

The morphology of nanofibers was obtained using a Field Emission Scanning Electron Microscope (FESEM, TESCAN MIRA 3) with fracture surfaces sputtered with gold.

\subsubsection{Fourier-transform infrared spectroscopy (FT-IR)}

FT-IR spectra in the region of $600-4000 \mathrm{~cm}^{-1}$ for pure carvedilol and selected formulations, were obtained by a Shimadzu IR-Prestige-21 FTIR spectrometer coupled with a horizontal Golden Gate MKII single-reflection ATR system (Specac, Kent, UK) and equipped with a Zn Se lens.

\subsubsection{Differential scanning calorimetry (DSC)}

Differential scanning calorimetry (DSC) was applied in order to determine thermal properties of carvedilol and selected formulations. Thermal properties were examined at temperatures ranging from room temperature up to $150^{\circ} \mathrm{C}$ on Mettler-Toledo AG, Analytical, Switzerland, DSC 1 under a dry nitrogen atmosphere (flow rate: $100 \mathrm{~cm}^{3} \mathrm{~min}^{-1}$ ) at a heating rate of $10{ }^{\circ} \mathrm{C} \mathrm{min}^{-1}$. The sample masses were between $7.3 \mathrm{mg}$ and $11.5 \mathrm{mg}$.

The degree of crystallinity, $\alpha$, of the polymer was calculated from $\Delta H_{\mathrm{m}}$, the heats of melting measured per gram of PEO according to the following equation

$\alpha=\frac{\Delta H}{\Delta H_{m}^{0}}$

where $\Delta H^{\circ}$ is $213.7 \mathrm{~J} / \mathrm{g}$ and presents the heat of melting per gram of $100 \%$ crystalline PEO (Grkovic et al., 2015).

\subsubsection{In vitro drug release studies}

Rotating paddle apparatus (Erweka DT70, Germany) was used, at rotating speed of $50 \mathrm{rpm} .900 \mathrm{ml}$ of $0.1 \mathrm{M} \mathrm{HCl}$ was used as dissolution medium, at $37^{\circ} \mathrm{C}$. Samples of $4 \mathrm{ml}$ were taken from medium at fixed time intervals $(10,20,30,45,60,90$ and $120 \mathrm{~min})$. All samples were filtered through membrane filter. The concentration of carvedilol was determined with UV/VIS spectrophotometer Evolution 300 (Thermo Fisher Scientific, Cambridge, UK). The results were shown as medium value of three repeated measurements for each sample. The weight of every sample was such that every sample has $25 \mathrm{mg}$ of carvedilol.

In order to assess an increase in dissolution rate of carvedilol from nanofibers and films, pure carvedilol $(25 \mathrm{mg})$ and immediate release tablets of carvedilol (Milenol ${ }^{\circledR}$, tablets, $25 \mathrm{mg}$, Hemofarm A.D, Serbia) were compared with developed formulations. Drug release profiles were compared using similarity factor (f2) (Moore and Flanner, 1996).

\section{Results and discussion}

\subsection{Carvedilol assay in nanofibers and films}

The results of carvedilol assay in nanofibers and films are shown in Table 2. It can be observed that the content of carvedilol in oral films is in accordance with the declared assay, regardless of the type of solvent used (over 92.8\%). In nanofibers made by electrospinning, when water was used as solvent (F4, F5), high amount of carvedilol was detected (92.4-98.4\%). However, when mixture of ethanol and water

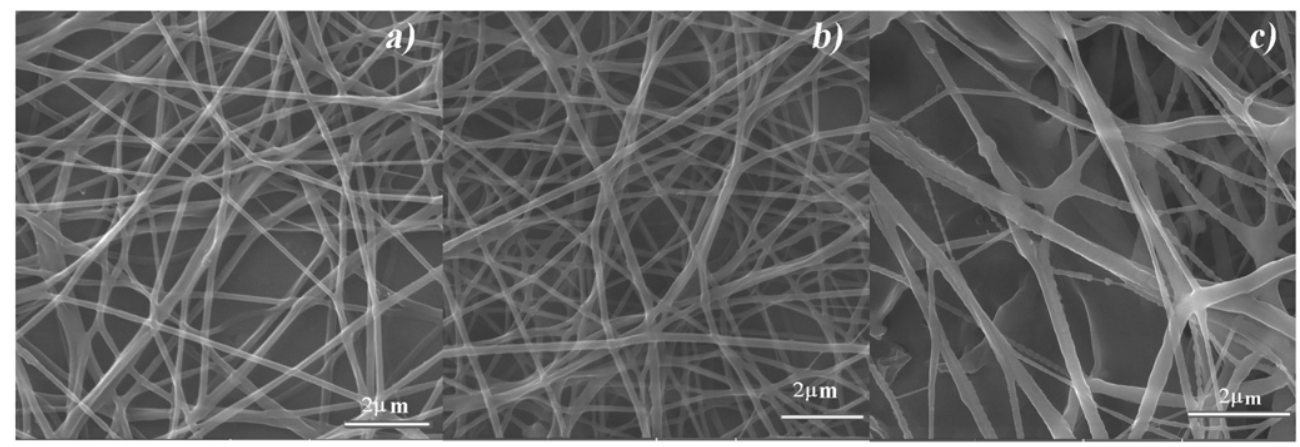

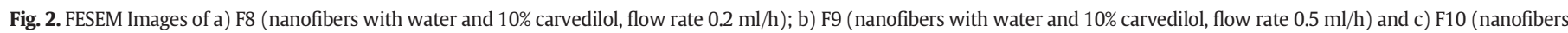
with water and $10 \%$ carvedilol, flow rate $1 \mathrm{ml} / \mathrm{h}$ ). 

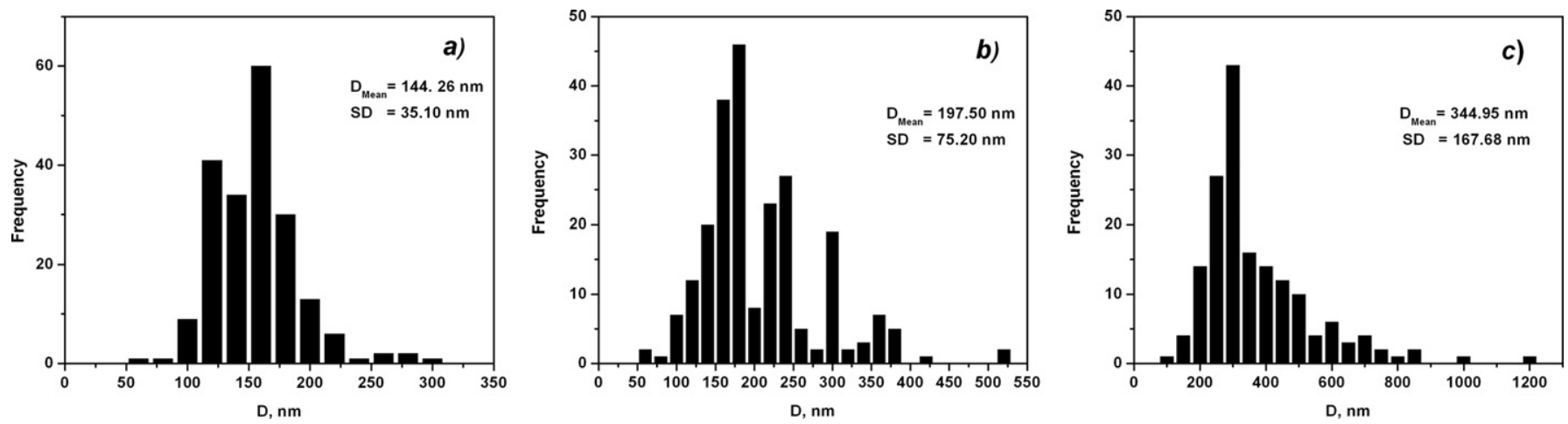

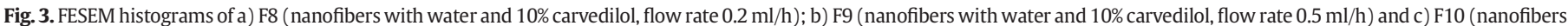
with water and $10 \%$ carvedilol, flow rate $1 \mathrm{ml} / \mathrm{h}$ ).

was used as solvent (F6, F7), significantly lower assay of carvedilol was measured (45.7-47\%). In the case, the evaporation of ethanol was faster, and part of carvedilol was precipitated on collector, not incorporated in fibers.

Based on obtained results, the water was selected as the most suitable solvent. In following experiments (F8, F9, F10), water was used as solvent, with increase in collector size, which provided nanofibers with high carvedilol assay (86.32-90.24\%).

Carvedilol assay in nanofibers prepared with PEO, with a mixture of water and ethanol was similar as in the study about nanofibers of carvedilol described in literature and prepared with polycaprolactone where it was $86-100 \%$. However, the degree of drug incapsulation into films was significantly higher (over 92.8) compared to carvedilol films, where it was 74-87\% (Potrč et al., 2015).

\subsection{Field Emission Scanning Electron Microscope (FESEM)}

Fig. 2 and Fig. 3 show images of F8, F9 and F10 nanofibers with fiber diameter distribution based on FESEM imaging (Other figures and histograms are presented in Supplementary data). Dimensions and diameter of fibers depend on the viscosity of solutions and process parameters of electrospinning - applied voltage, flow rate, collector size and distance between the needle and collector. It can be seen on FESEM images that, for different type of solvent used, fibers with different diameters are obtained. Obtained fibers have uniform diameter, but, due to instability of the process in case F5 drops and bubbles are formed into fibers (See Fig. 2 in Supplementary data).
FESEM Image of F4 (Fig. 1 in Supplementary data), which was prepared from water solution, shows that produced fibers have medium diameter of $132 \mathrm{~nm}$, and declared carvedilol content. FESEM image of formulation produced by electrospinning shows that F5 (Fig. 2 in Supplementary data) has fibers with medium diameter of $161 \mathrm{~nm}$. Droplets and beads are also present, due to nonstationary conditions, so this method can be characterized as a combination of electrospinning electrospraying. However, carvedilol was retained in these beads, resulting in high carvedilol loading in formulations, but uneven drug distribution.

FESEM images of formulations F6 and F7 (Figs. 3 and 4 in Supplementary data), produced from ethanol/water mixture, in order to obtain finer fibers with smaller diameter, show that obtained fibers have medium diameter of $172 \mathrm{~nm}$ and about 50\% reduced carvedilol content (45.7\%; 47\%), due to accelerated evaporation of ethanol and part of carvedilol was precipitated on collector. Figs. 3 and 4 in Supplementary data show drug agglomeration beyond fibers.

Figs. 2 and 3 show FESEM images of nanofiber formulations F8, F9, and F10. Produced fibers have medium diameter $144 \mathrm{~nm}, 197 \mathrm{~nm}$ and $345 \mathrm{~nm}$, respectively, with high carvedilol content. It can be observed that obtained fibers have uniform diameter (without droplets and beads), with the homogenous distribution of carvedilol (without agglomeration beyond fibers). An increase in the diameter of the nanofiber according to an increased flow rate was also observed in other studies (Zong et al., 2002; Bhardwaj and Kundu, 2010).

During the electrospinning process, when the polymer jet is ejected and accelerated toward the collector, the solvent evaporates rapidly as

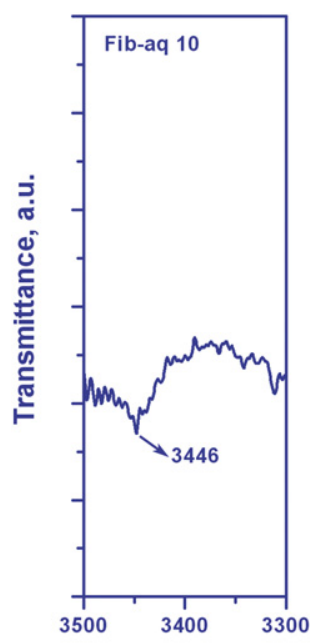

Wavenumber, $\mathrm{cm}^{-1}$
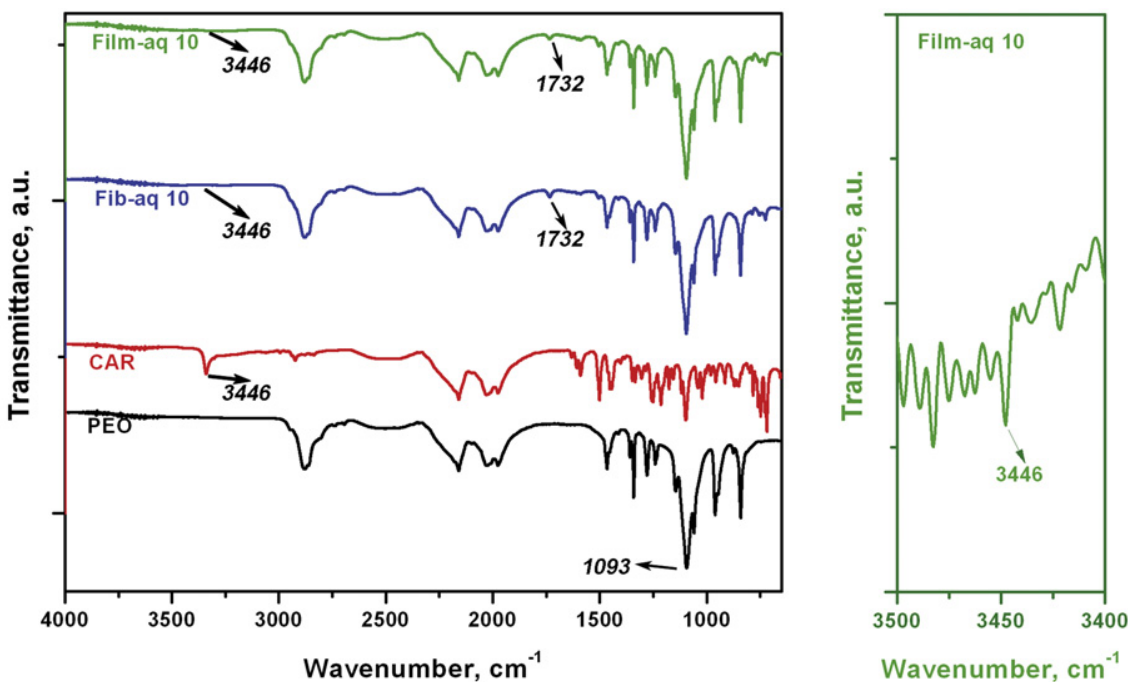

Fig. 4. FT-IR of PEO, carvedilol, film (formulation F2 (water, 10\% carvedilol (Film-aq-10))) and fibers (formulation F10 (water, $10 \%$ carvedilol (Fib-aq-10))) with carvedilol. 
the high surface area jet travels to the target. As an outcome of rapid evaporation of solvent, non-woven filament mats are formed rapidly, and this leads to a decrease in drug mobility. When solvent evaporation is complete, drug molecules are often "frozen" in the polymer fibers matrix. So, it was important to define electrospinning process parameters to obtain the smooth fiber with dimensions capable to carry the molecules of drugs, with no precipitation out of fibers (Yu et al., 2010). This condition was achieved in production of formulation F8, F9, and F10. In this case the homogeneity of samples is good.

\subsection{Fourier-transform infrared spectroscopy (FT-IR)}

In the FT-IR spectra of pure PEO the peak at $2886 \mathrm{~cm}^{-1}$ observed attributed to $\mathrm{C}-\mathrm{H}$ stretching (Fig. 4). The band at $1461 \mathrm{~cm}^{-1}$ is assigned to $\mathrm{CH}_{2}$ scissoring mode, while the peaks $1367 \mathrm{~cm}^{-1}$ and $1338 \mathrm{~cm}^{-1} \mathrm{CH}_{2}$ belonged to wagging mode. Also, the band at $1277 \mathrm{~cm}^{-1}$ was belonged to $\mathrm{CH}_{2}$ twisting mode, and for $\mathrm{C}-\mathrm{O}-\mathrm{C}$ stretching mode the peak was observed at $1093 \mathrm{~cm}^{-1}$. The peak at $956 \mathrm{~cm}^{-1}$ was representative for $\mathrm{CH}_{2}$ rocking and vibration mode, and at $841 \mathrm{~cm}^{-1}$ for $\mathrm{CH}_{2}$ rocking (Gondaliya et al., 2011).

An FT-IR spectrum of pure carvedilol showed the peaks $3446 \mathrm{~cm}^{-1}$ (N-H, stretching), $2926 \mathrm{~cm}^{-1}\left(\mathrm{C}-\mathrm{H}\right.$, stretching), $2842.60 \mathrm{~cm}^{-1}$ (C-H, stretching) and $1094 \mathrm{~cm}^{-1}$ ( $\mathrm{C}-\mathrm{O}$, stretching). These peaks can be considered as characteristic peaks of carvedilol (Dewan et al., 2012; Bal et al., 2013; Sharma, 2013).

The FT-IR spectra for film and nanofibers also have those bands and revealed that there no interaction between carvedilol, Polysorbate 80 and PEO. The peak at $1732 \mathrm{~cm}^{-1}$ could be attributed to the carbonyl group of Polysorbate 80.

\subsection{Differential scanning calorimetry (DSC)}

The results of DSC analysis of pure carvedilol, PEO and PEO/carvedilol films and fibers from different solution are presented in Fig. 5a. The melting peak of crystalline carvedilol was obtained at $117^{\circ} \mathrm{C}$. This peak was not detected in films and fiber samples, suggesting the amorphous form of carvedilol, which was dissolved and there was not there as crystalline phase (Balogh et al., 2015a, 2015b; Gao et al., 2005). Evaporation of solvent in electrospinnig processes are completed in very short time. So, there was no time for the drug molecules to form crystal lattices, and thus the drug complexed with the polymer on a molecular scale.

For PEO (as received) the melting point was at $71.34{ }^{\circ} \mathrm{C}$ (Ibrahim and Johan, 2012; Meka et al., 2014). Melting peaks of films and fibers with added carvedilol to PEO solutions was slightly changed due influence of carvedilol and surfactant Polysorbate 80 (Girdthep et al., 2011).

DSC curves of films with PEO polymers in different solvents (water or water/ethanol), both without and with Polysorbate 80, are shown on Fig. 5b. From this diagram it could be seen that $T_{m}$ of pure PEO after solution in water and mixing solvents is higher than for PEO powder (Table 3, Fig. 5). After that, added Polysorbate 80 as surfactant was acting as plasticizer and $\mathrm{T}_{\mathrm{m}}$ was decreased. It is a well-established and proven fact that a lamellar crystal is the fundamental structural form by which polymers most generally crystallize, a feature true for the vast majority of semicrystalline polymers crystallized from the bulk (i.e. from solution or from melt). Crystallinity of PEO was influenced with solvents and Polysorbate 80 too. The rigorous decrease in $\alpha$ from solution and Polysorbate 80 was the followed because the nucleation and growth of crystal was suppressed by difficult ordering of molecules dividing and oriented in hydrophobic - hydrophilic solvatation manner.

Crystallinity of polymers, represented by degree of crystallinity $(\alpha)$, is ratio of amount of crystalline region in polymer structure with respect to amorphous content. In crystal region polymers have ordered structure and molecules are arranged in a regular, periodic manner., while in the amorphous region the structure is quite random. The degree of crystallinity has a big influence on mechanical properties, density, transparency, diffusion and hence solubility. Degrees of crystallinity for PEO powder (as received), films F2 and F3, as well as fibers F5 and F6 are presented in Table 3. Crystallinity of PEO obtained from dispersions was decreased because there was a difficulty of PEO molecules to order in crystal with presence of solvent molecules. Also, with the adding of Polysorbate 80 in solution the conditions for crystallization of PEO was changed. It is obvious that degree of crystallinity of the films is higher than for electrospun fibers. During electrospinnig, the molecules that are aligned along the fiber axis have less time to realign themselves, leading to less favorable packing. For most semi-crystalline polymers, the stretched chains under high elongation rate do not get enough time to form crystalline lamellae, which yields lower crystallinity. Hence, the crystallinity in the fibers is thereby influenced by the rate of solvent evaporation (Baji et al., 2010; Huang et al., 2003; Greiner and Wendorff, 2007). Degrees of crystallinity for fibers (and films) with carvedilol from the aqueous solution are lower compared to fibers prepared with ethanol/water solutions.
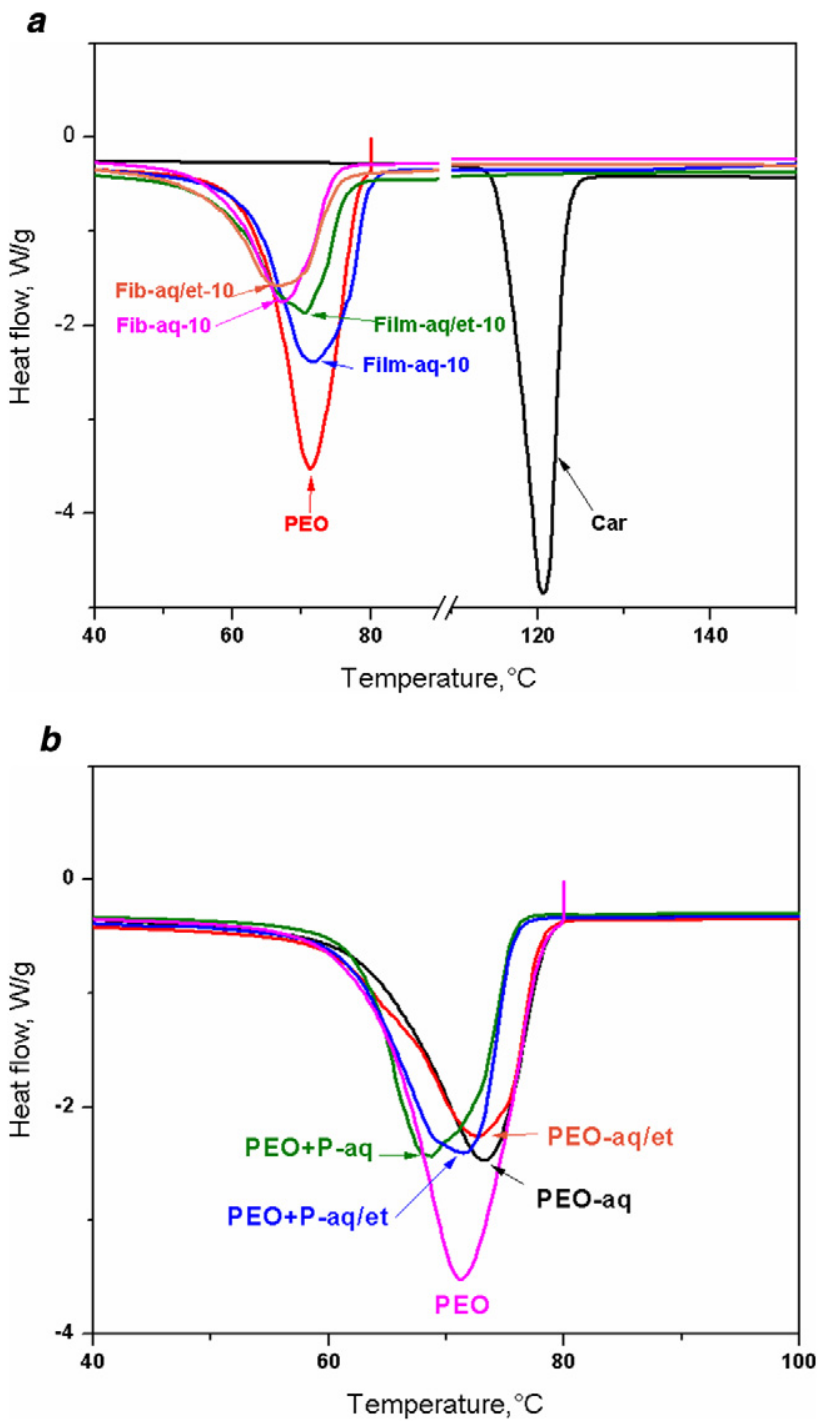

Fig. 5. a) DSC curves of PEO, carvedilol (Car), film (formulation F2 (water, 10\% carvedilo (Film-aq-10)) and formulation F3 (water/ethanol, 10\% (Film-aq/et-10))) and fibers (formulation F10 (water, 10\% carvedilol (Fib-aq-10)) and formulation F6 (water/ ethanol, $10 \%$ carvedilol declared, $4.57 \%$ assay (Fib-aq/et-10))). b) DSC curves of films: PEO in water (PEO-aq); PEO in mixture water/ethanol (PEO-aq/et), PEO in water with Polysorbate 80 (PEO + P-aq); PEO in mixture water/ethanol with Polysorbate 80 (PEO + P-aq/et). 
Table 3

Results of DSC analysis: melting point $\left(\mathrm{T}_{\mathrm{m}}\right)$ and degree of crystallinity $(\alpha)$.

\begin{tabular}{lll}
\hline Formulation & $\mathrm{Tm},{ }^{\circ} \mathrm{C}$ & $\alpha, \%$ \\
\hline Carvedilol & 120.68 & \\
PEO & 71.34 & 68.33 \\
F2 - film-aqueous solution 10\% & 71.71 & 38.94 \\
F3 - film- aqueous/ethanol solution 10\% & 70.48 & 44.56 \\
F5 - fibers - aqueous solution 10\% & 66.77 & 26.82 \\
F6 - fibers - aqueous/ethanol solution 10\% ${ }^{\mathrm{a}}$ & 68.00 & 38.67 \\
PEO-film aqueous solution & 73.20 & 19.74 \\
PEO-film aqueous/ethanol solution & 72.59 & 17.04 \\
PEO + Polysorbat 80-film aqueous solution & 68.75 & 16.24 \\
PEO + Polysorbat 80-film aqueous/ethanol solution & 71.46 & 13.11 \\
\hline
\end{tabular}

a $10 \%$ carvedilol declared ( $4.57 \%$ assay).

\subsection{In vitro drug release}

Carvedilol release from nanofibers and films is presented in Table 4. It can be observed from the results that significant increase in dissolution rate of carvedilol was obtained from all formulations, compared to pure carvedilol, and that it was similar to the dissolution profile of commercial immediate release carvedilol tablets. From all formulations, except F7, more than $80 \%$ of carvedilol was released in 30 min.

Comparing dissolution profiles and calculating similarity factors ( $f 2$ ) it was observed that dissolution profiles from all formulations are significantly different from dissolution profiles of pure carvedilol $(f 2<50)$. The percentage of carvedilol from the prepared nanofibers was significantly higher, compared to nanofibers of carvedilol prepared with polycaprolactone (Potrč et al., 2015). Potrč et al. have formulated nanofibers from which 15-30\% carvedilol has been created in $1 \mathrm{~h}$, whereas we achieved a dissolution of over $90 \%$ of carvedilol in $1 \mathrm{~h}$ (Potrč et al., 2015).

Comparing dissolution profiles of formulations F1-F3 with different amount of carvedilol in the film ( $5 \%$ or $10 \%$ ), it was observed that, when water was used as solvent (F1 and F2), dissolution profiles are significantly different ( $f 2-38.86$ ). This probably occurs due to low solubility of carvedilol in water. As a result, with increase in amount of carvedilol (in formulations where water was used as a solvent), dissolution rate of drug decreases. Nanofibers prepared with water, with different amount of carvedilol (F4 and F5), shown the same trend as films, and significant difference in dissolution rate was observed ( $f 2$ - 37.36). The highest dissolution rate was achieved with nanofibers with lower drug loading, probably for the same reason which was described at film formulations. When comparing different types of solvents used in formulation of oral films, significant difference in dissolution rate of carvedilol was observed at highest carvedilol assay in formulation - formulations F2 and F3 ( $f 2$ - 46.07). This probably occurs because carvedilol can be fully dissolved only in mixture of ethanol and water with Polysorbat 80 and PEO, becoming instantly available for dissolution from the formulation.

Comparing drug dissolution profiles from electrospun nanofibers and oral films made with water as solvent, using smaller size collectors were compared, it was not observed significant difference in dissolution rate of carvedilol, regardless the amount of drug in formulations (F1 and
F4 (f2 - 66.18); F2 and F5 ( $f 2$ - 60.04)). Changing the collector size, significant difference in dissolution rate of carvedilol was observed, when oral films and nanofibers prepared with water as solvent, and with $10 \%$ of carvedilol in formulation. Similarity factors were $35.09,36.37$, 39.64 for formulations F2 and F8; F2 and F9; F2 and F10, respectively. In this case, the higher dissolution rate was achieved from nanofibers. A lower percentage of drug dissolutions from films compared to fibers correlates with the results described in literature (Kenawy et al., 2002; Potrč et al., 2015). Differences in dissolution rate of carvedilol, made with changes in collector size, are probably the consequence of nanofibers diameter change, which was confirmed with FESEM analysis. Change in collector surface area, when using water as solvent, and with flow rate at $1 \mathrm{ml} / \mathrm{h}$ (F5 and F10), results in significant differences in dissolution rate of carvedilol, where the higher dissolution rate was achieved when bigger collectors were used ( $f 2$ - 17.87). The higher dissolution rate of carvedilol probably was a consequence of differences in size and structure of nanofibers, also more uniform distribution of drug, which was confirmed by FESEM analysis. It was not observed any significant difference in dissolution rate of carvedilol when mixture of water and ethanol was used as solvent, depending on flow rate used during preparation (F6 and F7 (f2 - 78.96)). Also, in the case of higher surface area of collector, increasing of the flow rate do not affect the dissolution rate of carvedilol (F8 and F9 ( $f 2$ - 73.22); F8 and F10 (f2 - 69.51); F9 and F10 $(f 2-70.23))$. During preparation of nanofibers flow rate can be increased, in order to achieve higher income, while preserving dissolution rate of carvedilol.

In F8, F9 and F10 fibers, significant improvement in dissolution rate was achieved, compared to F2 film formulation and F5 nanofiber formulation. This is probably the consequence of the same carvedilol content and it's uniform distribution in F8, F9 and F10 fibers. This can be observed on FESEM images. Therefore, after contact with dissolution medium carvedilol becomes immediately available for dissolution. F5 formulation has beads, and therefore uneven distribution of carvedilol, so the drug is not available for dissolution immediately after contact with dissolution medium. In oral film formulations, certain time is needed for dissolution of carvedilol, due to slower film disintegration and slower diffusion of the medium. Based on this comparison, it can be concluded that with increase in collector size, diameter of fibers increases, the distribution of carvedilol is more uniform, without beads, all of which leads to increase in dissolution rate of carvedilol.

\section{Conclusion}

Prepared films and nanofibers with carvedilol significantly increase the dissolution rate of carvedilol. FT-IR analysis of obtained samples shows that interaction between polymer and drug did not occur. DSC analysis revealed the dissolution of carvedilol in PEO and change of $\mathrm{T}_{\mathrm{m}}$ of PEO according formulation. Also, it was revealed that degree of PEO crystallinity of fibers was lower than films, because of faster solvent evaporation. FESEM analysis and content determination of drug in nanofibers show that, when mixture of ethanol and water is used as solvent, significant loss of carvedilol content from nanofibers occurs. Based

Table 4

Percent of carvedilol release from nanofibers and films, immediate release tablets and pure carvedilol.

\begin{tabular}{|c|c|c|c|c|c|c|c|c|c|c|c|c|}
\hline \multirow{3}{*}{ Time $(\min )$} & \multicolumn{12}{|c|}{ Percent of carvedilol release } \\
\hline & \multicolumn{3}{|l|}{ Films } & \multicolumn{7}{|c|}{ Nanofibers } & \multirow[t]{2}{*}{ Carvedilol } & \multirow[t]{2}{*}{ Tablets } \\
\hline & $\mathrm{F} 1$ & $\mathrm{~F} 2$ & F3 & $\mathrm{F} 4$ & F5 & F6 & F7 & F8 & F9 & F10 & & \\
\hline 10 & 68.27 & 36.57 & 63.47 & 67.19 & 28.21 & 43.68 & 43.88 & 79.80 & 76.45 & 72.00 & 18.32 & 64.56 \\
\hline 20 & 88.86 & 59.04 & 75.18 & 78.80 & 59.59 & 68.66 & 67.18 & 88.20 & 87.57 & 82.08 & 27.54 & 80.33 \\
\hline 30 & 92.32 & 85.16 & 83.96 & 88.35 & 80.91 & 80.25 & 76.34 & 88.90 & 88.27 & 86.40 & 30.13 & 83.46 \\
\hline 45 & 92.76 & 92.60 & 91.20 & 93.28 & 82.00 & 85.93 & 82.02 & 91.00 & 88.96 & 89.28 & 34.91 & 87.75 \\
\hline 60 & 93.75 & 94.20 & 92.05 & 96.06 & 87.98 & 91.47 & 88.99 & 93.81 & 90.35 & 93.89 & 37.12 & 88.69 \\
\hline 90 & 93.88 & 94.21 & 92.39 & 97.70 & 88.60 & 93.56 & 91.94 & 96.63 & 91.74 & 96.48 & 41.20 & 90.12 \\
\hline 120 & 94.59 & 94.24 & 92.58 & 98.12 & 94.27 & 95.13 & 94.88 & 98.01 & 93.13 & 96.75 & 42.02 & 92.69 \\
\hline
\end{tabular}


on aforementioned, it can be concluded that mixture of ethanol and water is not suitable as solvent for nanofibers production by electrospinning method. Significant differences between dissolution rate of carvedilol from the films and nanofibers were not observed, when smaller size collectors were used. When bigger collectors were used, significant difference in dissolution rate of carvedilol was observed, wherein the higher dissolution rate was achieved from nanofibers formulations.

This study is of exceptional significance in the impact assessment of process and formulation parameters on the characteristics of nanofibers produced by electrospinning process. Formulation F10 showed the fastest drug release, due to satisfactory release rate of carvedilol (86.40\% in $30 \mathrm{~min}$ ) as well as high drug loading (86.32\%), uniform carvedilol distribution inside the fibers, satisfactory fiber dimensions, together with high flow rate, which all lead to high income. The nanofibers formulated on this way in future should be defined into final pharmaceutical form (tablet or capsule), in order to increase bioavailability of carvedilol compared to the pure drug.

\section{Acknowledgements}

This work was done under the projects No. TR 34007 and TR 34011, supported by the Ministry of Education, Science and Technological Development, Republic of Serbia.

\section{Appendix A. Supplementary data}

Supplementary data to this article can be found online at http://dx. doi.org/10.1016/j.ejps.2017.02.006.

\section{References}

Agarwal, S., Wendorff, J.H., Greiner, A., 2008. Use of electrospinning technique for biomedical applications. Polymer 49, 5603-5621.

Baji, A., Mai, Y.W., Wong, S.C., Abtahi, M., Chen, P., 2010. Electrospinning of polymer nanofibers: effects on oriented morphology, structures and tensile properties. Compos. Sci. Technol. 70, 703-718.

Bal, T., Sengupta, S., Murthy, P.N., 2013. Formulation and evaluation of carvedilol microcapsules using Eudargit NE30D and sodium alginate. Braz. J. Pharm. Sci. 49 (4), 889-901.

Balogh, A., Drávavölgyi, G., Faragó, K., Farkas, A., Vigh, T., Sóti, P.L., Wagner, I., Madarász, J., Pataki, H., Marosi, G., Nagy, Z.K., 2014. Plasticized DrugLoaded melt electrospun polymer Mats: characterization, thermal degradation, and release kinetics. J. Pharm. Sci. 103 (4), 1278-1287.

Balogh, A., Cselkó, R., Démuth, B., Verreck, G., Mensch, J., Marosi, G., Nagy, Z., 2015a. Alternating current electrospinning for preparation of fibrous drug delivery systems. Int. J. Pharm. 495 (1), 75-80.

Balogh, A., Farkas, B., Faragó, K., Farkas, A., Wagner, I., Vanassche, I., Verreck, G., Nagy, Z.K., Marosi, G., 2015b. Melt-blown and electrospun drug-loaded polymer fiber Mats for dissolution enhancement: a comparative study. J. Pharm. Sci. 104 (5), 1767-1776.

Bertoncelj, V., Pelipenko, J., Kristl, J., Jeras, M., Cukjati, M., Kocbek, P., 2014. Development and bioevaluation of nanofibers with blood-derived growth factors for dermal wound healing. Eur. J. Pharm. Biopharm. 88 (1), 64-74.

Bhardwaj, N., Kundu, S.C., 2010. Electrospinning: a fascinating fiber fabrication technique. Biotechnol. Adv. 28 (3), 325-347.

Buschle-Diller, G., Cooper, J., Xie, Z., Wu, Y., Waldrup, J., Ren, X., 2007. Release of antibiotics from electrospun bicomponent fibers. Cellulose 14, 553-562.

Chew, S.Y., Wen, J., Yim, E.K.F., Leong, K.W., 2005. Sustained release of proteins from electrospun biodegradable fibers. Biomacromolecules 6 (4), 2017-2024.

Cui, W., Li, X., Zhu, X., Yu, G., Zhou, S., Weng, J., 2006. Investigation of drug release and matrix degradation of electrospun poly(dl-lactide) fibers with paracetanol inoculation. Biomacromolecules 7 (5), 1623-1629.

Dewan, I., Hossain, A., Ashraful, I., 2012. Formulation and evaluation of solid dispersions of carvedilol, a poorly water soluble drug by using different polymers. Int. J. Res. Pharm. Chem. 2 (3), 585-893.

Dukali, R., Radovic, I., Stojanovic, D., Sevic, D., Radojevic, V., Jocic, D., Aleksic, R., 2014 Electrospinning of the laser dye rhodamine B-doped poly(methyl methacrylate) nanofibers. J. Serb. Chem. Soc. 79 (7), 867-880.

Gao, W., Yu Bai, Y., Chen, E., Zhou, Q., 2005. Crystallization and melting of poly(ethylene oxide) confined in nanostructured particles with cross-linked shells of polybutadiene. Chin. J. Polym. Sci. 23 (3), 275-284.

Girdthep, S., Punyodom, W., Molloy, R., Channuan, W., 2011. Effect of Tween 80 on the Mechanical and Thermal Properties of Solution-Cast Blends of Poly(lactic acid) and Cellulose Acetate Butyrate Films, International Conference on Chemistry and Chemical Process IPCBEE. 10. IACSIT Press, Singapore.
Gondaliya, N., Kanchan, D.K., Sharma, P., Joge, P., 2011. Structural and conductivity studies of polyethylene oxide - silver triflate polymer electrolyte system. Mat. Sci. Apl. 2, 1639-1643.

Greiner, A., Wendorff, J.H., 2007. Electrospinning: a fascinating method for the preparation of ultrathin fibers. Angew. Chem. Int. Ed. 46, 5670-5703.

Grkovic, M., Stojanovic, D.B., Kojovic, A., Strnad, S., Kreze, T., Aleksic, R., Uskokovic, P.S. 2015. Keratin-polyethylene oxide bio-nanocomposites reinforced with ultrasonically functionalized graphen. RSC Adv. 5, 91280-91287.

Hammouda, B., 2006. Solvation characteristics of a model water-soluble polymer. J. Polym. Sci. B Polym. Phys. 44, 3195-3199.

Huang, Z.M., Zhang, Y.Z., Kotaki, M., Ramakrishna, S., 2003. A review on polymer nanofibers by electrospinning and their applications in nanocomposites. Compos. Sci. Technol. 63, 2223-2253.

Hunley, M.T., Long, T.E., 2008. Electrospinning functional nanoscale fibers: a perspective for the future. Polym. Int. 57, 385-389.

Ibrahim, S., Johan, M.R., 2012. Thermolysis and conductivity studies of poly(ethylene oxide) (PEO) based polymer electrolytes doped with carbon nanotube. Int. J. Electrochem. Sci. 7, 2596-2615.

Kalimuthu, S., Yadav, A.V., 2009. Formulation and evaluation of carvedilol loaded Eudragit E 100 nanoparticles. Int. J. Pharm. Tech. Res. 1 (2), 179-183.

Kenawy, El-R, Bowlin, G.L., Mansfield, K., Layman, J., Simpson, D.G., Sanders, E.H., Wnek, G.E., 2002. Release of tetracycline hydrochloride from electrospun poly(ethyleneco-vinylacetate), poly(lactic acid), and a blend. J. Control. Release 81, 57-64.

Lee, S.N., Poudel, B.K., Tran, T.H., Marasini, N., Pradhan, R., Lee, Y.I., Lee, D.W., Woo, J.S. Choi, H.G., Yong, C.S., Kim, J.O., 2013. A novel surface-attached carvedilol solid dispersion with enhanced solubility and dissolution arch. Pharm. Res. 36, 79-85.

Löbenberg, R., Amidon, G.L., 2000. Modern bioavailability, bioequivalence and biopharmaceutics classification system. New scientific approaches to international regulatory standards. Eur. J. Pharm. Biopharm. 50, 3-12.

Meinel, A.J., Germershaus, O., Luhmann, T., Merkle, H.P., Meinel, L., 2012. Electrospun matrices for localized drug delivery: current technologies and selected biomedical applications. Eur. J. Pharm. Biopharm. 81, 1-13.

Meka, V.S., Wee Liang, V.H., Dharmalingham, S.R., Sheshala, R., Gorajana, A., 2014. Preparation and in vitro characterization of non-effervescent floating drug delivery system of poorly soluble drug, carvedilol phosphate. Acta Pharma. 64, 485-494.

Moore, J.W., Flanner, H.H., 1996. Mathematical comparison of curves with an emphasis on in vitro dissolution profiles. Pharm. Technol. 20 (6), 64-74.

Nagy, Z.K., Balogh, A., Drávavölgyi, G., Ferguson, J., Pataki, H., Vajna, B., Marosi, G., 2013. Solvent-free melt electrospinning for preparation of fast dissolving drug delivery system and comparison with solvent-based electrospun and melt extruded systems. J. Pharm. Sci. 102 (2), 508-517.

Okuda, T., Tominaga, K., Kidoaki, S., 2009. Time-programmed dual release formulation by multilayered drug-loaded nanober meshes. J. Control. Release 143 (2), 258-264.

Potrč, T., Baumgartner, S., Roškar, R., Planinšek, O., Lavrič, Z., Kristl, J., Kocbek, P., 2015. Electrospun polycaprolactone nanofibers as a potential oromucosal delivery system for poorly water-soluble drugs. Eur. J. Pharm. Sci. 75, 101-113.

Rošic, R., Pelipenko, J., Kocbek, P., Baumgartner, S., Bešter-Rogač, M., Kristl, J., 2012. The role of rheology of polymer solutions in predicting nanofiber formation by electrospinning. Eur. Polym. J. 48, 1374-1384.

Sharma, A., 2013. Carvedilol- $\beta$-cyclodextrin Systems: Preparation, Characterization and in vitro Evaluation. Dhaka. Univ. J. Pharm. Sci. 12 (1), 51-58.

Siemann, U., 2005. Solvent cast technology - a versatile tool for thin film production. Progr. Colloid Polym. Sci. 130, 1-14.

Sill, T.J., von Recum, H.A., 2008. Electrospinning: applications in drug delivery and tissue engineering. Biomaterials 29, 1989-2006.

Taepaiboon, P., Rungsardthong, U., Supaphol, P., 2006. Drug-loaded electrospun mats of poly(vinyl alcohol) fibres and their release characteristics of four model drugs. Nanotechnology 17 (9), 2317-2329.

Xie, Z., Buschle-Diller, G., 2010. Electrospun poly(d,l-lactide) fibers for drug delivery: the influence of cosolvent and the mechanism of drug release. J. Appl. Polym. 115 (1), $1-8$.

Xie, J., Wang, C.-H., 2006. Electrospun micro- and nanofibers for sustained delivery of paclitaxel to treat C6 glioma in vitro. Pharm. Res. 23 (8), 1817-1826.

Yang, B., Wu, C., Ji, B., Ai, X., Kuang, X., Wu, M., Sun, M., Luo, C., He, Z., Sun, J., 2015. The biorelevant concentration of Tween 80 solution is a simple alternative medium to simulated fasted state intestinal fluid. RSC Adv. 5, 104846-104853.

Yu, D.G., Branford-White, C., White, K., Li, X.L., Zhu, L.M., 2010. Dissolution improvement of electrospun nanofiber-based solid dispersions for acetaminophen. AAPS PharmSciTech 11 (2), 809-817.

Yuvaraja, K., Khanam, J., 2014. Enhancement of carvedilol solubility by solid dispersion technique using cyclodextrins, water soluble polymers and hydroxyl acid. J. Pharm. Biomed. Anal. 96, 10-20.

Zamani, M., Morshed, M., Varshosaz, J., Jannesari, M., 2010. Controlled release of metronidazole benzoate from poly (- caprolactone) electrospun nanobers for periodontal diseases. Eur. J. Pharm. Biopharm. 75 (2), 179-185.

Zeng, J., Yang, L., Liang, Q., Zhang, X., Guan, H., Xu, X., Chen, X., Jin, X., 2005. Influence of the drug compatibility with polymer solution on the release kinetics of electrospun fiber formulation. J. Control. Release 105 (1-2), 43-51.

Zong, X., Kim, K., Fang, D., Ran, S., Hsiao, B.S., Chu, B., 2002. Structure and process relationship of electrospun bioabsorbable nanofiber membranes. Polymer 43 (16), 4403-4412. 